\title{
WOLF-RAYET STARS IN LOCAL GROUP GIANT HII REGIONS
}

\section{LAURENT DRISSEN}

\author{
Space Telescope Science Institute \\ 3700 San Martin Drive \\ Baltimore, MD 21218
}

\begin{abstract}
We present the results of our search for WR stars in three of M33's most massive giant HII regions, namely NGC 604, NGC 595 and NGC 592. A few of the putative "superluminous" WR stars are shown to be crowded multiple systems. We also compare the WR populations of the most massive GHRs in the Local Group. WN stars are 5 times more numerous on the average than WC stars in these large complexes. The variations among GHR WR populations are discussed in the context of metallicity and age differences.
\end{abstract}

\section{Introduction}

Giant HII regions (GHRs) appear to be the most intense sites of star formation known in normal galaxies, and they offer the unique opportunity to study massive stars at their birthplace. Apart from 30 Dor in the LMC, the most massive GHRs in the Local Group, namely NGC 604 and NGC 595, are located in M33. The presence of WR stars in these regions is well known, but some uncertainties remain. We present our observations in section 2 and 3 , and we compare the WR population of the best studied Local Group GHRs in section 4.

\section{Observations}

Narrow-band images of NGC 604, NGC 595 and NGC 592 were obtained with an RCA CCD detector attached to the Mont Mégantic 1.6m telescope. A set of three filters was used to detect WR stars:

$4780 / 65\left(\lambda_{c}=4780 \AA ; \mathrm{FWHM}=65 \AA\right)$; reference continuum $4686 / 35$; detect He II 4686 in WNs $4650 / 29$; detect CIII 4650 in WCs, NIII 4641 in WNs.

The reference continuum image was subtracted from the on-line image, after scaling in such a way that bright stars outside the nebula disappear completely. Details of the observations and reductions are presented elsewhere (Drissen, Moffat and Shara 1990, hereafter DMS).

\section{Results}

\subsection{NGC 604.}

With about $10^{6} \mathrm{M}_{\odot}$ of HII, NGC 604 is an order of magnitude more massive and more luminous than any known HII region in our Galaxy. D'Odorico and Rosa (1981) were 


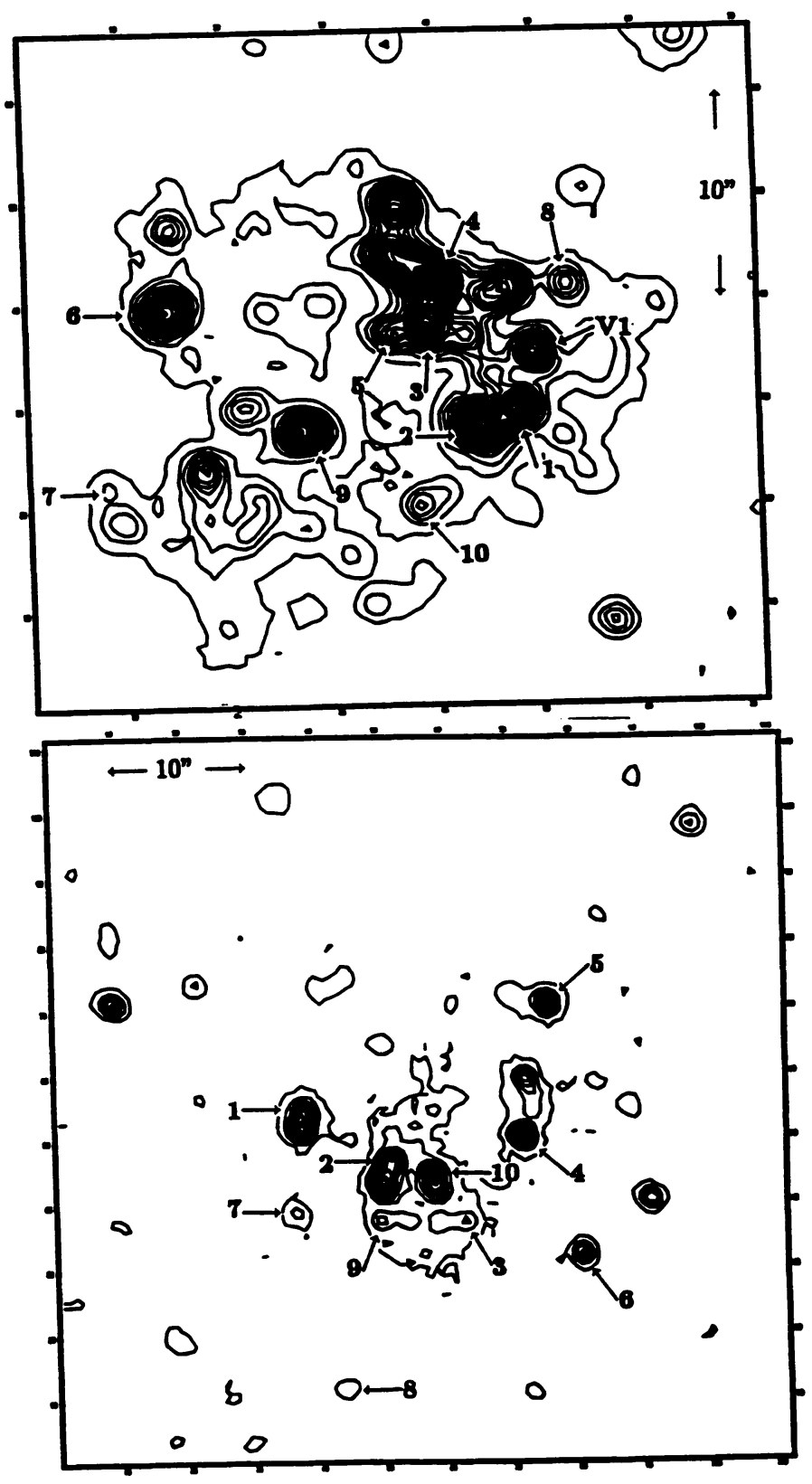

Figure 1. Isophotes of the on-line $4686 \AA$ image of NGC 604 (top) and NGC 595 (bottom), showing the positions of WR candidates. Star V1 in NGC 604 is variable (see DMS). North is at the top, east to the left. 
line flux in each object is consistent with that of a single Galactic WR star (DMS), we assume that only one WR star is present at each WR position.

Table 1 presents statistics on the WR population among the best studied and most massive GHRs in the Local Group. The total mass in OB stars has been derived by Kennicutt (1984) from the $\mathrm{H} \alpha$ flux. Although the statistics are rather poor, a few points should be noted. First, the number ratio of WR to $\mathrm{O}$ stars, WR/O (obtained by direct counting in resolved GHRs or deduced from the global light output in M33), is much lower than unity. A burst of star formation thus does not necessarily imply a large WR/O ratio, as it has sometimes been claimed (D'Odorico and Rosa 1981). However, this ratio does not seem to be constant from one region to the next. This is particularly obvious when one compares NGC 604 with NGC 595: both regions contain the same number of WR stars, despite the factor of two difference in mass.

TABLE 1. Local Group Giant HII Regions

\begin{tabular}{cccccccc}
\hline \hline Region & Galaxy & $\begin{array}{c}M_{\mathrm{OB}} \\
\left(M_{\odot}\right)\end{array}$ & $N_{\mathrm{WR}}$ & $\begin{array}{c}N_{\mathrm{WR}} / M_{\mathrm{OB}} \\
\left(\times 10^{-4}\right)\end{array}$ & $N(\mathrm{WC})$ & $N(\mathrm{WNL})$ & {$[\mathrm{O} / \mathrm{H}]$} \\
\hline 30 Dor & LMC & 50000 & 25 & 5 & 5 & 17 & $8,41^{(1)}$ \\
NGC 604 & M33 & 15000 & $7-10$ & $5-7$ & 2 & $>4$ & $8,51^{(2)}$ \\
NGC 595 & M33 & 7000 & $8-10$ & $11-14$ & 1 & 7 & $8,44^{(2)}$ \\
NGC 3603 & MW & 5000 & $2-3$ & $4-6$ & 0 & 2 & $8,39^{(3)}$ \\
Carina & MW & 2000 & 3 & 15 & 0 & 3 & $8,62^{(1)}$ \\
NGC 346 & SMC & 2000 & 1 & 5 & 0 & 0 & $8.22^{(1)}$ \\
NGC 592 & M33 & 1000 & $2-3$ & $20-30$ & 0 & - & - \\
\hline
\end{tabular}

Notes: For 30 Dor, we included all WR stars within 150 pc from R136, and considered that 4 WNL stars compose R136 (Moffat et al. 1987). The total mass in OB stars is taken from Kennicutt (1984). References for [O/H]: (1) Shaver et al. 1983; (2) Vilchez et al. 1988; (3) Melnick et al. 1989.

Three factors can in principle affect the WR/O ratio in GHRs: metallicity, the slope of the IMF and the age of the burst. Both theoretically and observationally, metallicity has been shown to be the major factor controlling WR/O and WC/WN number ratios (Smith 1988, Maeder 1990; see however Massey 1990). However, [O/H] is very similar in NGC 604 and NGC 595. A flatter IMF could also increase the WR/O ratio, without affecting the WC/WN ratio as much (Maeder 1990). Although this hypothesis cannot be rejected, there is little, if any, evidence for changes in the slope of the IMF as a function of galactocentric distance in M33 (Freedman 1985), or between the field and starburst regions (Blaha and Humphreys 1989, Massey, Parker and Garmany 1989). Another parameter that could affect the WR/O ratio is the age of the burst. Time dependent synthetic WR/O ratios computed by Arnault, Kunth and Schild (1989) show that the WR/O ratio increases very rapidly a few Myrs after the burst, stays constant for a while (the actual duration depending on the metallicity) and decreases rapidly after that. Thus, two young starburst regions 
the first to discover WR stars in that region: spectroscopy of the brightest knots revealed the presence of WR characteristics in most of them. This led the authors to conclude that an unusually large number of WR stars $(\sim 50)$ were present in that nebula. Conti and Massey (1981, hereafter CM) have also detected WR emission lines in three objects. Assuming that these objects were single stars, they concluded that they were, like R136 in the core of 30 Dor, superluminous.

An isophote map of the on-line $4686 \AA$ image of NGC 604 is displayed in Figure 1 , indicating the position of our WR candidates. Stars nos. $1,2,3,4,6$ and 9 are spectroscopically confirmed WR stars (all but no. 1 being WNs; see CM, D'Odorico and Rosa 1981, DMS). Stars 1 and 2 were considered until now as a single WR star (CM11), but they are clearly resolved in our images. Moreover, star no. 2 is elongated and is very likely to be a composite object (see also Debray 1990). Another CM object, no. 12 in their list, is also resolved here into two components: nos. 3 $(\mathrm{WN}$ ) and 5 ( likely to be a WC). Detection levels of candidates 7, 8 and 10 are quite low and spectroscopy is an absolute requirement before considering them as genuine WRs. The absolute visual magnitude $\left(\mathrm{M}_{V}\right)$ of the brightest WR stars in NGC 604 is close to -7.7 (Debray 1990), about a magnitude fainter than what was previously thought.

\subsection{NGC 595.}

This region has received much less attention than NGC 604, although the presence of WR stars has been known since CM obtained spectra of 3 luminous stars in its core. Massey and Conti (1983) and Massey, Conti and Armandroff (1987) have spectroscopically confirmed three more WR stars, and Armandroff and Massey (1985) have detected three more candidates. An isophote map of the on-line $4686 \AA$ image of NGC 595 is presented in Figure 1. Two of the previous "superluminous" WR stars, nos. 1 (CM6) and 2 (CM5) are clearly resolved from adjacent bright stars. Their new estimated magnitudes are still quite high (-6.9 for no. 1 and -7.9 for no. 2), but no more than some WNL stars, e.g. as seen in 30 Dor (Moffat 1989). Lequeux, Meyssonier and Azzopardi (1987) have mentionned objects 4 and 5 in their objective prism survey. Star no. 3 is the only WC star in that region. Armandroff and Massey (1990) have recently obtained spectra of stars 4, 5 and 7 and classify them as WNL.

\subsection{NGC 592.}

This region is marginal compared to NGC 604 ( about 10 times less massive), but nevertheless contains a few WR stars. We have resolved another putative superluminous WR star (CM3) into two components, the fainter one being the WN star. Its previously estimated $M_{V}(-9)$ is now reduced to -7 . Another confirmed WN star (no. 2 in Massey, Conti and Armandroff 1987) is $20^{\prime \prime}$ to the west of CM3. We have also detected a new WR candidate, probably of WN type.

\section{THE WOLF-RAYET POPULATION OF GIANT HII REGIONS}

It is a difficult task to deduce the exact number of WR stars in each knot from our data alone, because a large number of abnormally weak emission-line Of/WR stars could mimic the appearance of a single, "normal" WR star, at least in the brightest knots. However, there is no need to postulate such an anomaly and since the emission- 
of slightly different ages can have very different $\mathrm{WR} / \mathrm{O}$ ratios.

The other important fact to notice from Table 1 is that all these GHRs are completely dominated by WN stars, most of them being of late (6-7) types. The global WC/WR ratio in these regions is $\sim 0.17$. The lack of WC stars in starburst "WR galaxies" has also been noticed (Vacca and Conti 1990). Metallicity certainly plays an important role in this trend. With the exception of the Carina nebula, all these regions have a low metal abundance (unfortunately, there is no GHR comparable to NGC 604 or 30 Dor in M31, where the metallicity is much higher). However, this does not explain the fact that 30 Dor contains mainly WNL stars while the rest of the LMC is dominated by WNE (Moffat et al. 1987). Models (Langer 1987; Maeder 1990) indicate that the duration of the WNL phase increases with the mass of the progenitor. In this context, the observations could be explained if the average mass of the WR progenitors in GHRs is higher than in the field, or that these regions are so young that only the most massive stars have evolved away from the main sequence.

Many points remain unsettled and there is an absolute need for high resolution imagery and high signal-to-noise spectroscopy of the most massive stars of the Local Group GHRs, as well as in larger starburst regions, such as the "hypergiant" HII regions in M101 where WR spectral features have been detected (D'Odorico, Rosa and Wampler 1983).

\section{References}

Armandroff, T. E. and Massey, P. 1985, Ap. J., 291, 685.

Armandroff, T. E. and Massey, P. 1990, this Symposium.

Arnault, P., Kunth, D. and Schild, H. 1989, Astron. Ap., 224, 73.

Blaha, C. and Humphreys, R. M. 1990, A. J., 98, 1598.

Conti, P. S. and Massey, P. 1981, Ap. J., 249, 471 (CM).

Debray, B. 1990, this Sympcsium.

D'Odorico, S. and Rosa, M. 1981, Ap. J., 248, 1015.

D'Odorico, S., Rosa, M. and Wampler, E. J. 1983, Astron. Ap. Sup., 53, 97.

Drissen, L., Moffat, A. F. J. and Shara, M. M. 1990, Ap. J., in press.

Freedman, W. L. 1985, Ap. J., 299, 74.

Kennicutt, R. C. 1984, Ap. J., 287, 116.

Langer, N. 1987, Astron. Ap., 171, L1.

Lequeux, J., Meyssonnier, N. and Azzopardi, M. 1987, Astron. Ap. Sup., 67, 169.

Maeder, A. 1990, Astron. Ap., in press.

Massey, P. 1990, this Symposium.

Massey, P. and Conti, P. S. 1983, Ap. J., 273, 576 (MC).

Massey, P., Conti, P. S. and Armandroff, T. E. 1987, A. J., 94, 1538.

Massey, P., Parker, J. W. and Garmany, C. D. 1989, A. J., 98, 1305.

Melnick, J., Tapia, M. and Terlevich, R. 1989, Astron. Ap., 213, 89.

Moffat, A. F. J. 1989, Ap. J., 347, 373.

Moffat, A. F. J. , Niemela, V. S., Phillips, M. M., Chu, Y.-H. and Seggewiss, W. 1987, Ap. J., 312, 612.

Shaver, P. A., Mc Gee, R. X., Newton, L. M., Danks, A. C. and Pottasch, S. R. 1983, M. N. R. A. S., 204, 53 .

Smith, L. F. 1988, Ap. J., 327, 128.

Vacca, W. D. and Conti, P. S. 1990, preprint. 


\section{DISCUSSION}

Vilchez: How would sequential star formation affect the WR/WC ratio?

Drissen: According to Arnault, Kunth and Schild (1989), the shorter the burst is, the higher the WR/O ratio would be. Sequential star formation would then decrease the $\mathrm{WR} / \mathrm{O}$ ratio.

Maeder: The aging in a starburst region can lead to large $\mathrm{WR} / \mathrm{O}$ ratios as you have shown. However, this is likely to apply only to regions limited in size. For large regions or galaxies an average star formation rate over the last million years is more likely.

Drissen: I agree that one should distinguish between the field stars and starburst regions. The WR/O and WC/WN ratios may be different in the two cases. However, a burst of star formation does not necessarily mean very high WR/O ratios (close to 1 ), as we can see in Local Group GHR.

Laurent Drissen

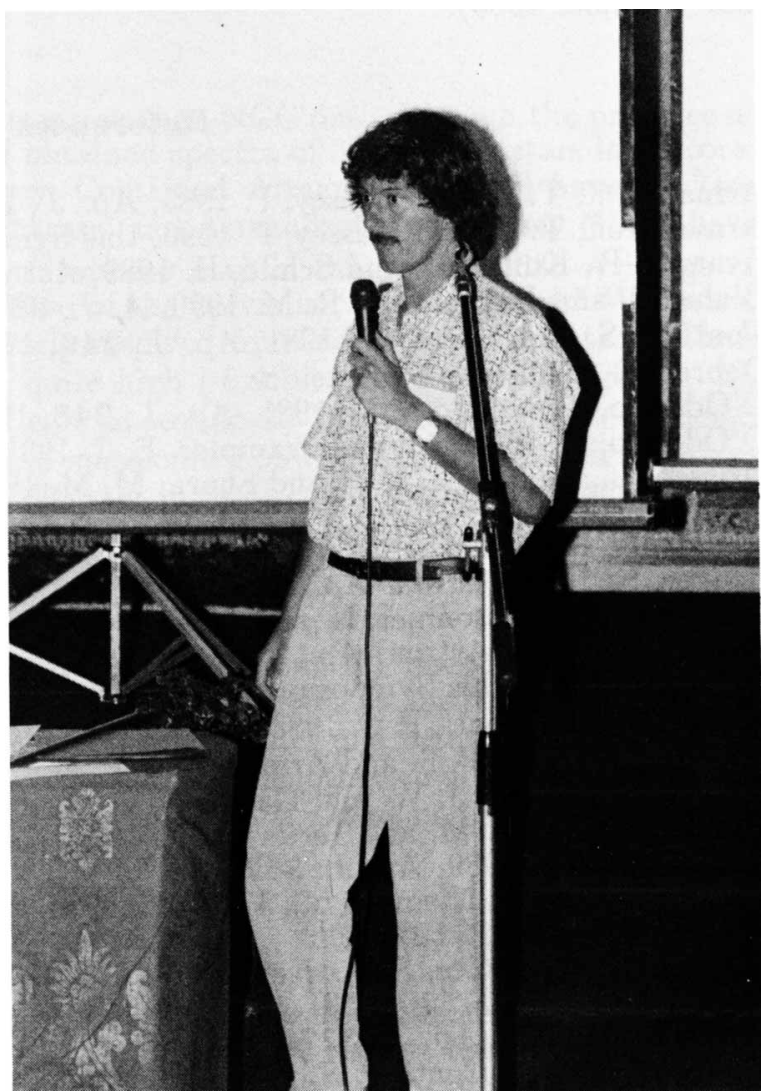

